\title{
Decorating loose defect-free hybrid selective layer on smooth intermediary:an effective way of forward osmosis membrane
}

\author{
Haiting Shi ${ }^{1}$, Qin Liu ${ }^{1}$, Wei Wang ${ }^{1}$, Mingjing Shan ${ }^{1}$, Kunyue Teng ${ }^{1}$, Hui Deng ${ }^{1}$, Zhiwei \\ $\mathrm{Xu}^{2}$, and Xiaoming Qian ${ }^{1}$ \\ ${ }^{1}$ Affiliation not available \\ ${ }^{2}$ Tiangong University
}

June 15, 2020

\begin{abstract}
A novel composite forward osmosis (FO) membrane was fabricated by introducing a chitosan (CS) sublayer between nanofibers and polyamide (PA) layer, which was beneficial to forming thin and defect-free PA layer and improved binding strength between substrate and selective layer. Results exhibited low reverse salt flux (0.73 gMH) while maintained high water flux (85.4 LMH), which were better than that in other reports. In order to further improve permeability of membranes, oxidized multiwall carbon nanotubes (OMWNTs) were added into PA layer based on the optimized sublayer. The crosslinking degree of PA layer was reduced, leading to loose PA layers, which promoted the increase of water flux. After modifying, water flux reached 96.9 LMH without sacrificing salt rejection. It was worth mentioning that PAN/CS composite FO membrane, which could adjust microstructure of support layer and selective layer respectively, may provide a promising way for preparing membranes with anticipated separation performance.
\end{abstract}

\section{Hosted file}

Main Document.doc available at https://authorea.com/users/333749/articles/459839-decoratingloose-defect-free-hybrid-selective-layer-on-smooth-intermediary-an-effective-way-offorward-osmosis-membrane

\section{Hosted file}

Table.doc available at https://authorea.com/users/333749/articles/459839-decorating-loosedefect-free-hybrid-selective-layer-on-smooth-intermediary-an-effective-way-of-forwardosmosis-membrane

\section{Hosted file}

Figure captions.docx available at https://authorea.com/users/333749/articles/459839decorating-loose-defect-free-hybrid-selective-layer-on-smooth-intermediary-an-effectiveway-of-forward-osmosis-membrane

\section{Hosted file}

Fig1.doc available at https://authorea.com/users/333749/articles/459839-decorating-loosedefect-free-hybrid-selective-layer-on-smooth-intermediary-an-effective-way-of-forwardosmosis-membrane

\section{Hosted file}


Fig2.doc available at https://authorea.com/users/333749/articles/459839-decorating-loosedefect-free-hybrid-selective-layer-on-smooth-intermediary-an-effective-way-of-forwardosmosis-membrane

\section{Hosted file}

Fig3.doc available at https://authorea.com/users/333749/articles/459839-decorating-loosedefect-free-hybrid-selective-layer-on-smooth-intermediary-an-effective-way-of-forwardosmosis-membrane

\section{Hosted file}

Fig4.doc available at https://authorea.com/users/333749/articles/459839-decorating-loosedefect-free-hybrid-selective-layer-on-smooth-intermediary-an-effective-way-of-forwardosmosis-membrane

\section{Hosted file}

Fig5.doc available at https://authorea.com/users/333749/articles/459839-decorating-loosedefect-free-hybrid-selective-layer-on-smooth-intermediary-an-effective-way-of-forwardosmosis-membrane 